\title{
Spectral Numerical Study of a Problem Governed by Navier-Stokes Equations, Influence of Rayleigh and Prandtl Numbers
}

\author{
E. El Guarmah ${ }^{1,2}$ and A. Cheddadi ${ }^{1 *}$ \\ ${ }^{1}$ EMI, Mohamed V University, Ibn Sina Str., POB 765 Agdal, Rabat, Morocco \\ ${ }^{2}$ Royal Air School, Mathematics and Informatics Department, BEFRA, Marrakech, Morocco
}

\begin{abstract}
We present in this work a numerical study of a problem governed by Navier-Stokes equations and heat equation. The mathematical problem under consideration is obtained by modelling the natural convection of an incompressible fluid, in laminar flow between two horizontal concentric coaxial cylinders, the temperature of the inner cylinder is supposed to be greater than the outer one. The numerical simulation of the flow is carried out by collocation-Legendre method. The influence of Prandtl and Rayleigh numbers is investigated.
\end{abstract}

Key words: Navier-Stokes equations, heat equation, collocation-Legendre method, Crank-Nicholson method

AMS subject classification: 76M22, 80M25

\section{Introduction}

Let us consider the following dimensionless vorticity-stream function formulation of the NavierStokes equations and heat equation, under the Boussinesq approximation $[4,8]$ :

$$
\begin{aligned}
& \operatorname{Pr} \Delta \omega-\operatorname{Ra} \operatorname{Pr}\left(\frac{\cos \theta}{r} \frac{\partial T}{\partial \theta}+\sin \theta \frac{\partial T}{\partial r}\right)=-\frac{1}{r}\left(\frac{\partial \Psi}{\partial \theta} \frac{\partial \omega}{\partial r}-\frac{\partial \Psi}{\partial r} \frac{\partial \omega}{\partial \theta}\right), \\
& \Delta T-\frac{1}{r}\left(\frac{\partial \Psi}{\partial \theta} \frac{\partial T}{\partial r}-\frac{\partial \Psi}{\partial r} \frac{\partial T}{\partial \theta}\right)=0, \quad \Delta \Psi=-\omega,
\end{aligned}
$$

*Corresponding author. E-mail: cheddadi@emi.ac.ma 
where $\Psi$ is the stream function, $\omega$ is the vorticity and $T$ is the temperature, $R a$ is the dimensionless Rayleigh number and $\operatorname{Pr}$ is the dimensionless Prandtl number. The problem under consideration is two-dimensional laminar convection in an incompressible fluid confined between two horizontal concentric coaxial cylinders, with inner and outer radii $r_{i}$ and $r_{e}$, respectively $[3,5,6,9]$. The inner cylinder is considered to be held at a uniform temperature $T_{i}$, and the outer cylinder at a uniform temperature $T_{e}$, with $T_{i}>T_{e}$.

A Fourier series expansion in the azimuthal direction is adopted and collocation-Legendre method is applied in the radial direction. We obtain a system of coupled Helmholtz equations. The Crank-Nicholson semi-implicit temporal scheme is used. The collocation-Legendre method proposed in this paper is restricted to those simplified geometries that can accommodate the special grid requirements. It may be particularly relevant to wall-bounded flows such as the present one, because more grid points are located in the region of strongest gradients near the walls. Detailed investigation of various aspects of spectral methods can be found in $[1,2,7,10,11,12]$.

\section{Results and discussion}

The numerical code is written in $C^{++}$. Numerical solutions are obtained for cylinders with a radius ratio $1.2 \leq R \leq 3$ and Rayleigh number up to $R a=10^{5}$. The results of the present study were found qualitatively and quantitatively valid when compared with experimental and numerical investigations carried out earlier.

\subsection{Influence of the Prandtl number}

We varied the Prandtl number $(P r)$ between 0.7 and $\infty$. The Rayleigh number was fixed at a value of $R a=10^{4}$ large enough to ensure strong convective flow behavior. Our main focus lied on the question how the Prandtl number affects the spatial structure of the flow. We also investigated the functional dependence of the Nusselt number ( $N u$, the rate of convective heat transport) on the value of the Prandtl number. For low Prandtl numbers, $\operatorname{Pr}<10$, convective heat transport is mainly due to a large scale convection cell. The effective heat transport rate $N u$ increases significantly with $\operatorname{Pr}$. In the high Prandtl number case, $\operatorname{Pr}>10, \mathrm{Nu}$ is nearly constant, see Figure 1. It will be noted that we find for $\operatorname{Pr}=\infty$ exactly the same results as in [3].

\subsection{Influence of the Rayleigh number}

Figures 2 and 3 show typical results obtained for Rayleigh numbers $R a=10^{3}$ and $R a=10^{4}$, with a radial ratio $R=1.6$ and $R=2$ respectively, and for different values of the collocation points number $M$ and the Fourier-series representation order $N$. The zero initial conditions induce a unicellular flow in a half annulus. The distortion of isotherms in Figure 3(b) reveals a strong convective motion inside the cavity. It is also noticed that the maximum heat transfer, indicated by closely spaced isotherms, is located at the top of the cavity for the outer cylinder and at the bottom 


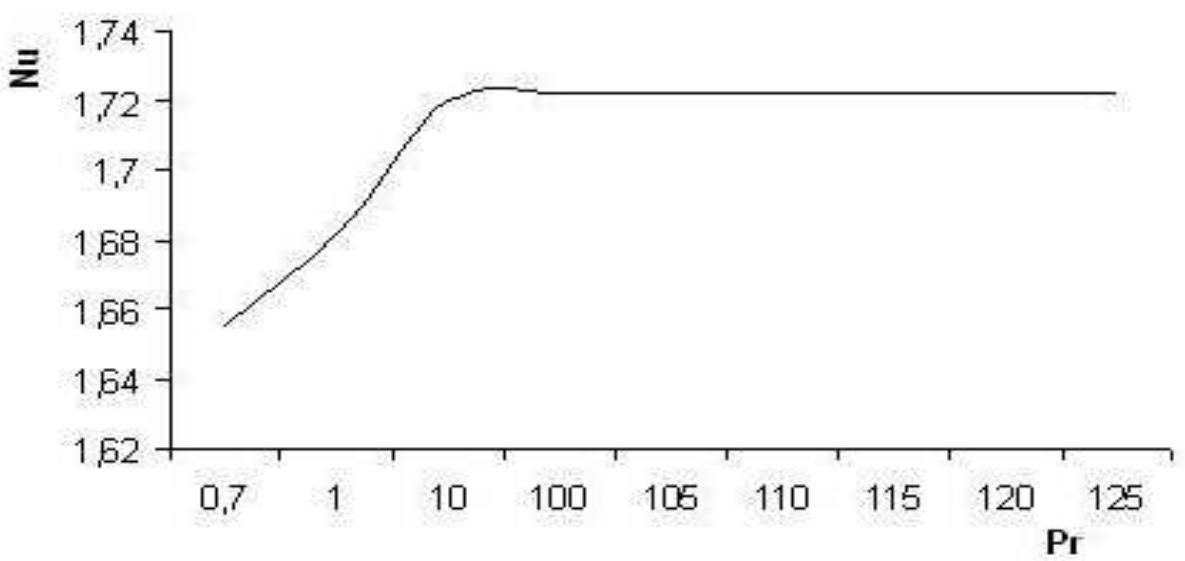

Figure 1: Variation of $N u$ in function of $\operatorname{Pr}$, for $R a=10^{4}, R=1.6 M=40$ and $N=20$.

for the inner one. Figures 4 and 5 show the variation with $R a$, of $N u$ and the maximal value of stream function $\Psi_{\max }$.
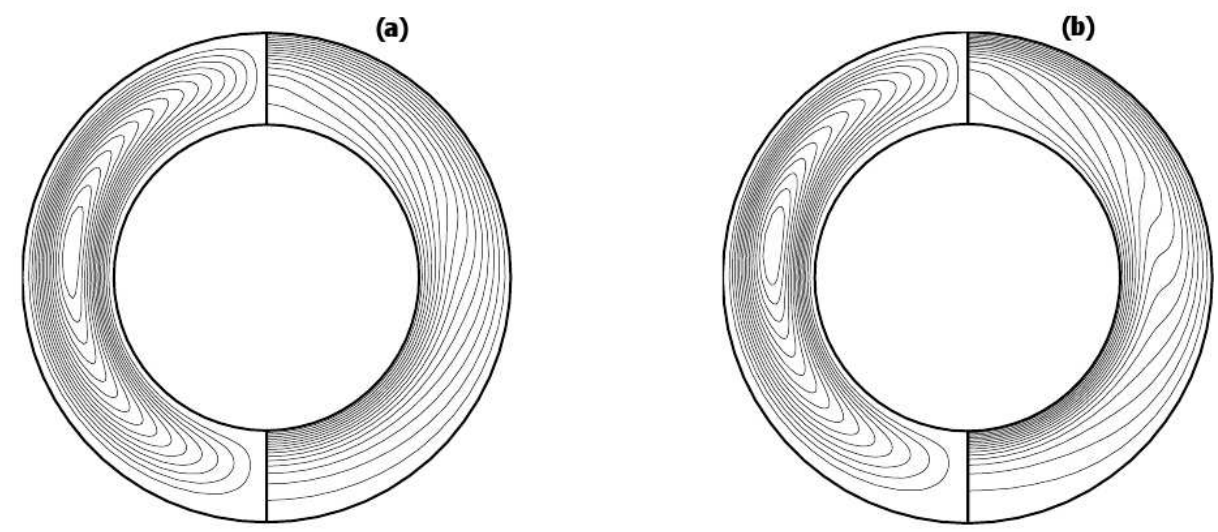

Figure 2: Isotherms and streamlines for $R=1.6$, (a) $R a=5 \times 10^{3}, M=45, N=45,(b)$ $R a=10^{4}, M=50, N=50$.

\section{Conclusion}

In this paper, the influence of the Prandtl and Rayleigh numbers on heat transfer in a problem modelled by Navier-Stokes equations coupled with heat equation in an annular cylindrical cavity is investigated. A Fourier series expansion in the azimuthal direction is adopted and collocationLegendre method is applied in the radial direction. The Crank-Nicholson semi-implicit temporal 

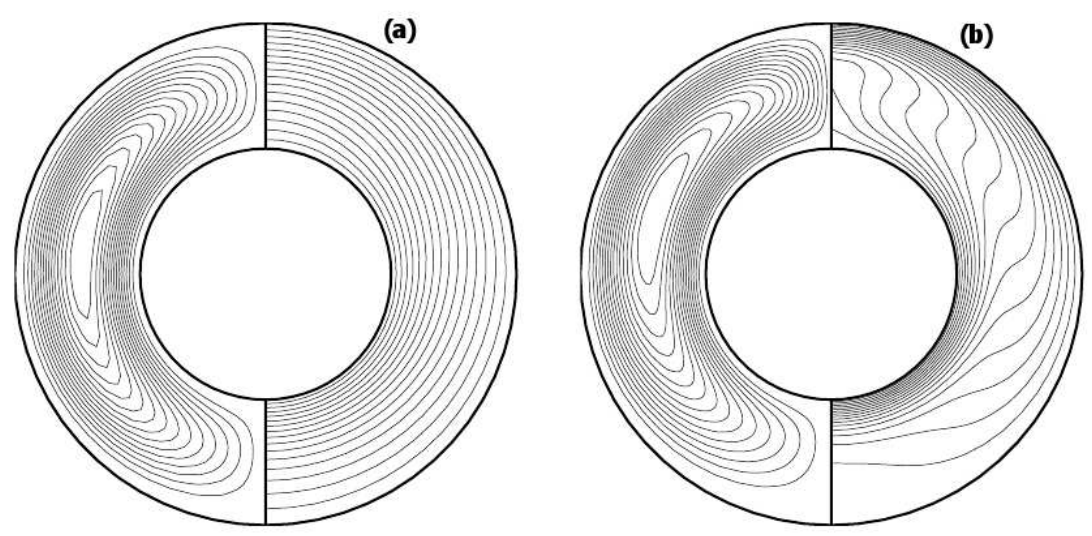

Figure 3: Isotherms and streamlines for $R=2$, (a) $R a=10^{3}, M=30, N=30$, (b) $R a=$ $10^{4}, M=50, N=50$.

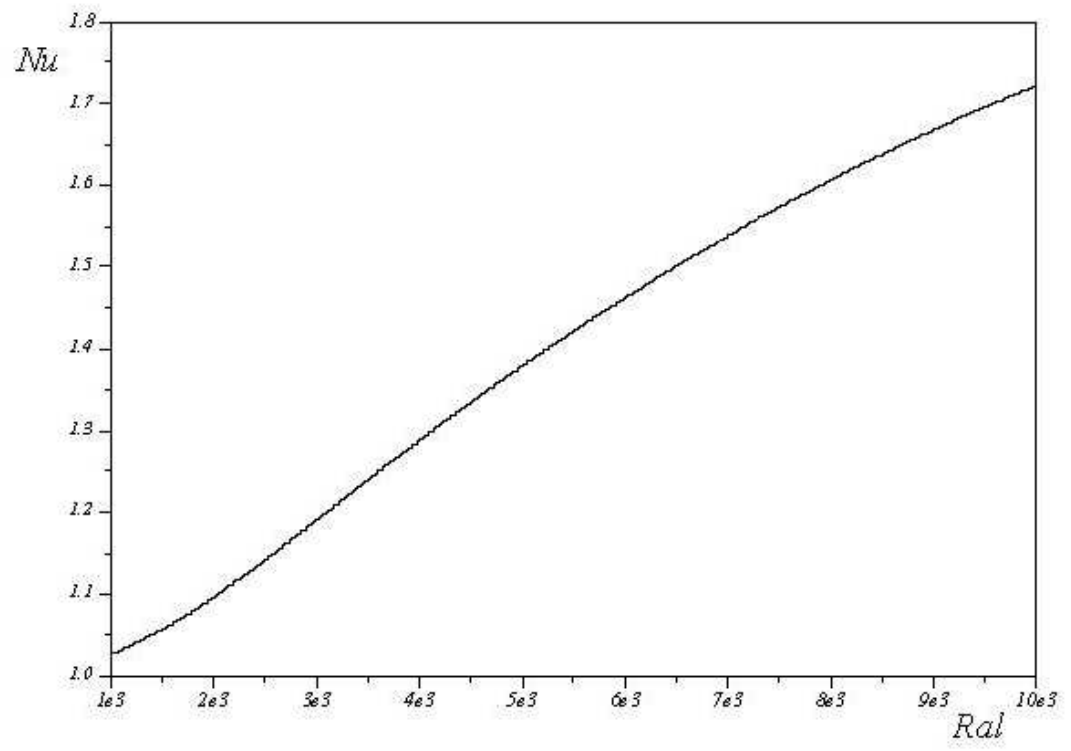

Figure 4: Profile $N u(R a)$ for $R=1.6, \operatorname{Pr}=0.7$ and $M=N=30$.

scheme is used. The results are in good agreement with available experimental data and other solutions in the literature.

\section{References}

[1] C. Bernardi, Y. Maday. Approximations spectrales de problèmes aux limites elliptiques. Springer-Verlag France, Paris, 1992. 


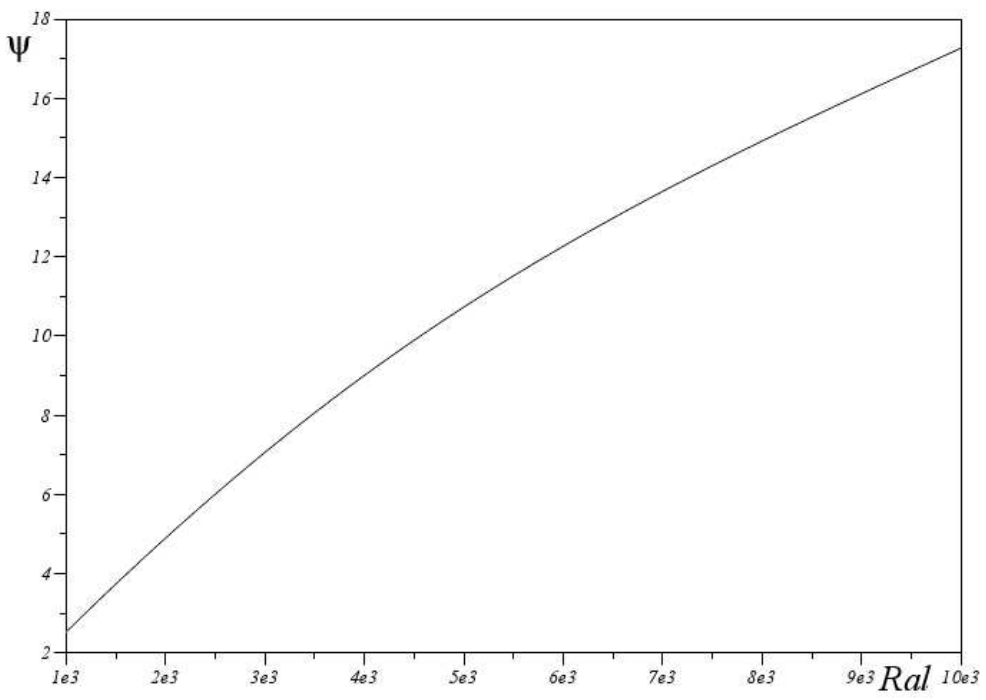

Figure 5: Profile $\Psi_{\max }(R a)$ for $R=1.6, \operatorname{Pr}=0.7$ and $M=N=30$.

[2] C. Canuto, M. Y. Hussaini, A. Quarteroni, T. Zang. Spectral methods in fluid dynamics. Springer, New York, 1988.

[3] A. Cheddadi, E. El Guarmah. Discrétisation spectrale d'un écoulement fluide Prandtl infini dans une géométrie annulaire cylindrique. PCN Journal, Physical and Chemical News, 30 (2006), 42-48.

[4] A. Cheddadi, E. El Guarmah. Influence of the collocation grid on a collocation-Chebyshev procedure for the numerical simulation of Navier-Stokes equations. AMSE Journal, Modelling and Simulation, Best of Book (2005), 127-136.

[5] A. Cheddadi, M. C. Charrier-Mojtabi, A. Mojtabi. Étude comparative de la convection naturelle dans des espaces annulaires cylindriques fluide et poreux: 1-écoulements bidimensionnels multicellulaires. 6ème Colloque Maghrébin sur les modèles numériques de l'ingénieur (C2MNI6), Tunis, 24-26 Novembre 1998.

[6] L. Crawford, R. Lemlich. Natural convection in horizontal concentric cylindrical annuli. IEC Fundamentals, 1 (1962), 260-264.

[7] B. Fornberg. A practical guide to pseudo spectral methods. Cambridge University Press, 1996.

[8] D. Gray, A. Giorgini. The Validity of the Boussinesq approximation for liquids and gases. Int. J. Heat Mass Transfer, 19 (1976), 545-551.

[9] T. H. Kuehn, R. H. Goldstein. An experimental and theoretical study of natural convection in the annulus between horizonatl concentric cylinders. J. Fluid, 74 (1976), 695-719. 
[10] R. Peyret. Introduction to spectral methods. Computational Fluid Dynamics, Lecture Series Von Karman, 1986-04.

[11] A. Quarteroni. Blending Fourier and Chebyshev interpolation. J. Approx. Theory, 51 (1987), 115-126.

[12] M. Uhlmann. The need for de-aliasing in a Chebyshev pseudo-spectral method. Num. Meth. for Fluid Dynamics, (1986), 463-475. 